\title{
Global function fields with many rational places over the quinary field. II
}

\author{
by
}

Harald Niederreiter (Wien) and Chaoping Xing (Singapore)

1. Introduction. Let $q$ be an arbitrary prime power and $K$ a global function field with full constant field $\mathbb{F}_{q}$, i.e., with $\mathbb{F}_{q}$ algebraically closed in $K$. We use the notation $K / \mathbb{F}_{q}$ if we want to emphasize the fact that $\mathbb{F}_{q}$ is the full constant field of $K$. By a rational place of $K$ we mean a place of $K$ of degree 1 . We write $g(K)$ for the genus of $K$ and $N(K)$ for the number of rational places of $K$. For fixed $g \geq 0$ and $q$ we put

$$
N_{q}(g)=\max N(K)
$$

where the maximum is extended over all global function fields $K / \mathbb{F}_{q}$ with $g(K)=g$. Equivalently, $N_{q}(g)$ is the maximum number of $\mathbb{F}_{q}$-rational points that a smooth, projective, absolutely irreducible algebraic curve over $\mathbb{F}_{q}$ of given genus $g$ can have. The calculation of $N_{q}(g)$ is a very difficult problem, so usually one has to be satisfied with bounds for $N_{q}(g)$. Upper bounds for $N_{q}(g)$ that improve on the classical Weil bound can be obtained by a method of Serre [15] (see also [16, Proposition V.3.4]).

Global function fields $K / \mathbb{F}_{q}$ of genus $g$ with many rational places, that is, with $N(K)$ reasonably close to $N_{q}(g)$ or to a known upper bound for $N_{q}(g)$, have received a lot of attention in the literature. We refer to Garcia and Stichtenoth [1], Niederreiter and Xing [10], [11], and van der Geer and van der Vlugt [17] for recent surveys of this subject. The construction of global function fields with many rational places, or equivalently of algebraic curves over $\mathbb{F}_{q}$ with many $\mathbb{F}_{q}$-rational points, is not only of great theoretical interest, but it is also important for applications in the theory of algebraicgeometry codes (see [13], [16]) and in recent constructions of low-discrepancy sequences (see [5], [9], [12]).

In the present paper we concentrate on the case $q=5$ and extend the list of constructions of global function fields $K / \mathbb{F}_{5}$ with many rational places in [6, Section 5] and [8]. The motivation for this is that the recent tables of

1991 Mathematics Subject Classification: 11G09, 11G20, 11R58, 14G15, $14 \mathrm{H} 05$. 
lower and upper bounds for $N_{q}(g)$ in [11] and [12] cover all genera $g \leq 50$, except in the case $q=5$ where they cover only the range $g \leq 22$. We now close this gap by providing constructions for $q=5$ and $23 \leq g \leq 50$, and in fact for many other values of the genus. A crucial role in this is played by a general construction principle based on Hilbert class fields.

In Section 2 we review some background on Hilbert class fields and narrow ray class extensions. Section 3 presents the general construction principle mentioned above and a list of examples for $q=5$ derived from this principle. Further examples for $q=5$ obtained by other methods are given in Section 4.

2. Background for the constructions. First we recall some pertinent facts about Hilbert class fields. A convenient reference for this topic is Rosen [14]. Let $F$ be a global function field with $N(F) \geq 1$ and distinguish a rational place $\infty$ of $F$. The Hilbert class field $H_{\infty}$ of $F$ with respect to $\infty$ is the maximal unramified abelian extension of $F$ (in a fixed separable closure of $F$ ) in which $\infty$ splits completely. The extension $H_{\infty} / F$ is finite and its Galois group is isomorphic to the fractional ideal class group $\operatorname{Pic}(A)$ of the ring $A$ of elements of $F$ that are regular outside $\infty$. In the case under consideration ( $\infty$ rational), $\operatorname{Pic}(A)$ is isomorphic to the $\operatorname{group} \operatorname{Div}^{0}(F)$ of divisor classes of $F$ of degree 0 . In particular, we have $\left[H_{\infty}: F\right]=h(F)$, the divisor class number of $F$. For each place $P$ of $F$ there is an associated Galois automorphism $\tau_{P} \in \operatorname{Gal}\left(H_{\infty} / F\right)$, and the Artin symbol of $P$ for the extension $H_{\infty} / F$ is equal to $\tau_{P}$. The place $P$ corresponds to the divisor class of $P-\operatorname{deg}(P) \infty$ in $\operatorname{Div}^{0}(F)$. There is also a standard identification between places of $F$ and prime ideals in $A$.

Next we collect some facts about narrow ray class extensions which can be found in [2, Section 7.5] and [4, Section 16]. Let $F=F / \mathbb{F}_{q}, \infty$, and $A$ be as above and let $\phi$ be a sign-normalized Drinfeld $A$-module of rank 1 . By [4, Section 15] we can assume that $\phi$ is defined over the Hilbert class field $H_{\infty}$, i.e., that for each $z \in A$ the $\mathbb{F}_{q}$-endomorphism $\phi_{z}$ is a polynomial in the Frobenius with coefficients from $H_{\infty}$. If $\bar{H}_{\infty}$ is a fixed algebraic closure of $H_{\infty}$ and $M$ a nonzero integral ideal in $A$, then we write $\Lambda_{M}$ for the $A$ submodule of $\bar{H}_{\infty}$ consisting of the $M$-division points. Let $E_{M}:=H_{\infty}\left(\Lambda_{M}\right)$ be the subfield of $\bar{H}_{\infty}$ generated over $H_{\infty}$ by all elements of $\Lambda_{M}$. Then $E_{M} / F$ is called the narrow ray class extension of $F$ with modulus $M$. The field $E_{M}$ is independent of the specific choice of the sign-normalized Drinfeld $A$-module $\phi$ of rank 1 . Furthermore, $E_{M} / F$ is a finite abelian extension with

$$
\operatorname{Gal}\left(E_{M} / F\right) \simeq \operatorname{Pic}_{M}(A):=\mathcal{I}_{M}(A) / \mathcal{P}_{M}(A),
$$

where $\mathcal{I}_{M}(A)$ is the group of fractional ideals of $A$ that are prime to $M$ and $\mathcal{P}_{M}(A)$ is the subgroup of principal fractional ideals that are generated by 
elements $z \in F$ with $z \equiv 1 \bmod M$ and $\operatorname{sgn}(z)=1$ (here sgn is the given sign function). We have $\operatorname{Gal}\left(E_{M} / H_{\infty}\right) \simeq(A / M)^{*}$, the group of units of the ring $A / M$. Thus, if $\Phi_{q}(M)$ denotes the order of the latter group, then

$$
\left[E_{M}: F\right]=\left|\operatorname{Pic}_{M}(A)\right|=h(F) \Phi_{q}(M) .
$$

If $M=Q^{n}$ with a nonzero prime ideal $Q$ in $A$ and $n \geq 1$, then

$$
\Phi_{q}\left(Q^{n}\right)=\left(q^{d}-1\right) q^{d(n-1)}
$$

where $d$ is the degree of the place of $F$ corresponding to $Q$. Again in this situation, $E_{M} / F$ is unramified away from $\infty$ and $Q$. Furthermore, the decomposition group (and also the ramification group) $D_{\infty}$ of $\infty$ in $E_{M} / F$ is the subgroup $D_{\infty}=\left\{c+M: c \in \mathbb{F}_{q}^{*}\right\}$ of $(A / M)^{*}$, and every place of $H_{\infty}$ lying over $Q$ is totally ramified in $E_{M} / H_{\infty}$.

In the special case where $F$ is the rational function field $\mathbb{F}_{q}(x)$ over $\mathbb{F}_{q}$, the theory of narrow ray class extensions reduces to that of cyclotomic function fields as developed by Hayes [3]. In this case it is customary to take for $\infty$ the unique pole of $x$ in $\mathbb{F}_{q}(x)$. We will use the convention that a monic irreducible polynomial $P$ over $\mathbb{F}_{q}$ is identified with the place of $\mathbb{F}_{q}(x)$ which is the unique zero of $P$, and we will denote this place also by $P$.

3. Examples from Hilbert class fields. We first establish a general construction principle for global function fields with many rational places that is based on Hilbert class fields.

TheOREM 1. Let $q$ be odd, let $S$ be a subset of $\mathbb{F}_{q}$, and put $n=|S|$. Choose a polynomial $f \in \mathbb{F}_{q}[x]$ such that $\operatorname{deg}(f)$ is odd, $f$ has no multiple roots, and $f(c)=0$ for all $c \in S$. For the global function field $F=\mathbb{F}_{q}(x, y)$ with $y^{2}=f(x)$, assume that its divisor class number $h(F)$ is divisible by $2^{n} m$ for some positive integer $m$. Then there exists a global function field $K / \mathbb{F}_{q}$ such that

$$
g(K)=\frac{h(F)}{2^{n+1} m}(\operatorname{deg}(f)-3)+1 \quad \text { and } \quad N(K) \geq \frac{(n+1) h(F)}{2^{n} m},
$$

with equality if $n=q$.

Proof. Note that $F$ is a Kummer extension of the rational function field $\mathbb{F}_{q}(x)$ with

$$
g(F)=\frac{1}{2}(\operatorname{deg}(f)-1)
$$

by [16, Example III.7.6]. For each $c \in S$ the place $x-c$ of $\mathbb{F}_{q}(x)$ is totally ramified in $F / \mathbb{F}_{q}(x)$, and so is the pole of $x$ in $\mathbb{F}_{q}(x)$. Let $\infty$ denote the unique place of $F$ lying over the pole of $x$ in $\mathbb{F}_{q}(x)$. For the principal divisor $(x-c)$ of $F$ we thus have

$$
(x-c)=2 P_{c}-2 \infty,
$$


where all $P_{c}, c \in S$, are rational places of $F$. Consequently, the divisor class of $P_{c}-\infty$ has order 1 or 2 in the group $\operatorname{Div}^{0}(F)$, and so the subgroup $J$ of $\operatorname{Div}^{0}(F)$ generated by the divisor classes of all $P_{c}-\infty, c \in S$, has order dividing $2^{n}$. It follows that there exists a subgroup of $G$ of $\operatorname{Div}^{0}(F)$ with $|G|=2^{n} m$ and $G \supseteq J$. Let $H_{\infty}$ be the Hilbert class field of $F$ with respect to the rational place $\infty$ and let $K$ be the subfield of the extension $H_{\infty} / F$ fixed by $G$, viewed as a subgroup of $\operatorname{Gal}\left(H_{\infty} / F\right)$. Then

$$
[K: F]=\frac{h(F)}{2^{n} m} .
$$

By construction, the places $\infty$ and $P_{c}, c \in S$, split completely in the extension $K / F$, and this yields the desired lower bound for $N(K)$. Furthermore, $K / F$ is an unramified extension, and so the formula for $g(K)$ follows immediately from the Hurwitz genus formula.

REMARK. It is obvious that there is an analog of Theorem 1 with base fields $F$ that are general Kummer extensions of $\mathbb{F}_{q}(x)$ with arbitrary $q$, but Theorem 1 is of sufficient generality for our purposes.

From now on we take $q=5$. In Table 1 we list examples of global function fields $K / \mathbb{F}_{5}$ with many rational places that are obtained from Theorem 1 . The table contains the following data: the value of the genus $g(K)$, the value or a lower bound for the number $N(K)$ of rational places, the values of $n$ and $m$, the polynomial $f(x)$, and the value of the divisor class number $h(F)$ of $F=\mathbb{F}_{5}(x, y)$ with $y^{2}=f(x)$. In the cases where the exact value of $N(K)$ is indicated, it can be obtained from Theorem 1 or by other simple arguments. The divisor class numbers $h(F)$ have been calculated by the standard method based on the results in [16, Section V.1] and with the help of the software package Mathematica. Table 1 contains entries for $g(K)=$ 15,19 , and 21 that improve on earlier examples in [8].

Table 1

\begin{tabular}{cccccc}
\hline$g(K)$ & $N(K)$ & $n$ & $m$ & $f(x)$ & $h(F)$ \\
\hline 15 & $=35$ & 4 & 1 & $x(x+1)(x+2)(x-1)\left(x^{3}+x^{2}+x-2\right)$ & 112 \\
19 & $\geq 45$ & 4 & 1 & $x(x+1)(x+2)(x-2)\left(x^{3}-2 x^{2}+2 x-2\right)$ & 144 \\
21 & $=50$ & 4 & 1 & $\left(x^{5}-x\right)\left(x^{2}-x+1\right)$ & 160 \\
23 & $=55$ & 4 & 1 & $x(x+1)(x+2)(x-1)\left(x^{3}+x^{2}-2 x+1\right)$ & 176 \\
24 & $=46$ & 1 & 1 & $x\left(x^{4}+x^{3}+2 x^{2}+x-2\right)$ & 46 \\
27 & $=52$ & 1 & 1 & $x(x-1)\left(x^{3}-x+2\right)$ & 52 \\
28 & $=54$ & 5 & 2 & $\left(x^{5}-x\right)\left(x^{2}-2 x-2\right)\left(x^{2}-2 x-1\right)$ & 576 \\
29 & $\geq 56$ & 3 & 1 & $x(x+1)(x+2)(x-1)\left(x^{3}+x^{2}+x-2\right)$ & 112 \\
30 & $=58$ & 1 & 1 & $x\left(x^{4}+x^{2}+2\right)$ & 58 \\
32 & $=62$ & 1 & 1 & $x\left(x^{4}+2 x^{3}-2 x^{2}-2 x+2\right)$ & 62 \\
\hline
\end{tabular}


Table 1 (cont.)

\begin{tabular}{|c|c|c|c|c|c|}
\hline$g(K)$ & $N(K)$ & $n$ & $m$ & $f(x)$ & $h(F)$ \\
\hline 35 & $\geq 68$ & 3 & 1 & $x(x+1)(x+2)\left(x^{4}+x^{2}-2 x-2\right)$ & 136 \\
\hline 37 & $=72$ & 3 & 1 & $x(x+1)(x+2)\left(x^{4}-2 x-1\right)$ & 144 \\
\hline 39 & $=76$ & 3 & 1 & $x(x+1)(x+2)\left(x^{4}+x^{3}-2 x^{2}+2 x+1\right)$ & 152 \\
\hline 40 & $=65$ & 4 & 3 & $x(x+1)(x+2)(x-2)\left(x^{5}+2 x^{2}-2 x+1\right)$ & 624 \\
\hline 41 & $=80$ & 3 & 1 & $x(x+1)(x+2)\left(x^{4}+x-1\right)$ & 160 \\
\hline 43 & $=84$ & 3 & 1 & $x(x+1)(x+2)\left(x^{4}-2 x^{2}-2\right)$ & 168 \\
\hline 45 & $=88$ & 3 & 1 & $x(x+1)(x+2)\left(x^{4}+2 x^{2}+2 x+1\right)$ & 176 \\
\hline 46 & $\geq 75$ & 4 & 4 & $x(x+1)(x+2)(x-2)\left(x^{3}-x^{2}-x+2\right)\left(x^{2}+x+2\right)$ & 960 \\
\hline 47 & $=92$ & 3 & 1 & $x(x+1)(x+2)\left(x^{4}-2 x^{2}-x-2\right)$ & 184 \\
\hline 49 & $=96$ & 3 & 1 & $x(x+1)(x+2)\left(x^{4}+x^{3}+2 x^{2}+2\right)$ & 192 \\
\hline 52 & $=102$ & 5 & 1 & $\left(x^{5}-x\right)\left(x^{4}+x^{2}+2 x+2\right)$ & 544 \\
\hline 53 & $=104$ & 3 & 1 & $x(x+1)(x+2)\left(x^{2}+x+2\right)\left(x^{2}-x+1\right)$ & 208 \\
\hline 55 & $=108$ & 3 & 1 & $x(x+1)(x+2)\left(x^{4}+x^{2}+2 x+2\right)$ & 216 \\
\hline 57 & $=112$ & 3 & 1 & $x(x+1)(x+2)\left(x^{4}-2 x^{2}+x+1\right)$ & 224 \\
\hline 58 & $\geq 95$ & 4 & 3 & $x(x+1)(x+2)(x-2)\left(x^{5}+2 x^{2}+1\right)$ & 912 \\
\hline 61 & $=120$ & 5 & 1 & $\left(x^{5}-x\right)\left(x^{4}+x^{2}+2\right)$ & 640 \\
\hline 64 & $\geq 105$ & 4 & 2 & $x(x+1)(x+2)(x-2)\left(x^{2}+x+1\right)\left(x^{3}-x^{2}-2\right)$ & 672 \\
\hline 67 & $=132$ & 3 & 1 & $x(x+1)(x+2)\left(x^{4}+x^{3}+x-2\right)$ & 264 \\
\hline 70 & $\geq 115$ & 4 & 2 & $x(x+1)(x+2)(x-2)\left(x^{2}+2 x-1\right)\left(x^{3}-2 x^{2}-1\right)$ & 736 \\
\hline 76 & $=150$ & 5 & 1 & $\left(x^{5}-x\right)\left(x^{4}+2\right)$ & 800 \\
\hline 85 & $=140$ & 4 & 1 & $x(x+1)(x+2)(x-2)\left(x^{3}-x^{2}-1\right)\left(x^{2}+x+1\right)$ & 448 \\
\hline 91 & $\geq 150$ & 4 & 2 & $x(x+1)(x+2)(x-2)\left(x^{3}-x^{2}-x+2\right)\left(x^{2}+x+2\right)$ & 960 \\
\hline 94 & $=155$ & 4 & 1 & $x(x+1)(x+2)(x-2)\left(x^{5}+x^{2}-2 x+2\right)$ & 496 \\
\hline 97 & $=160$ & 4 & 1 & $x(x+1)(x+2)(x-2)\left(x^{2}+x+2\right)\left(x^{3}-x^{2}-x-1\right)$ & 512 \\
\hline 100 & $=165$ & 4 & 1 & $x(x+1)(x+2)(x-2)\left(x^{5}+x^{2}+2 x-1\right)$ & 528 \\
\hline 103 & $\geq 170$ & 4 & 1 & $\left(x^{5}-x\right)\left(x^{4}+x^{2}+2 x+2\right)$ & 544 \\
\hline 109 & $\geq 180$ & 4 & 1 & $\left(x^{5}-x\right)\left(x^{2}-2 x-2\right)\left(x^{2}-2 x-1\right)$ & 576 \\
\hline 118 & $=195$ & 4 & 1 & $x(x+1)(x+2)(x-2)\left(x^{5}+2 x^{2}-2 x+1\right)$ & 624 \\
\hline 121 & $=200$ & 4 & 1 & $\left(x^{5}-x\right)\left(x^{4}+x^{2}+2\right)$ & 640 \\
\hline 127 & $=210$ & 4 & 1 & $x(x+1)(x+2)(x-2)\left(x^{2}+x+1\right)\left(x^{3}-x^{2}-2\right)$ & 672 \\
\hline 139 & $=230$ & 4 & 1 & $x(x+1)(x+2)(x-2)\left(x^{2}+2 x-1\right)\left(x^{3}-2 x^{2}-1\right)$ & 736 \\
\hline 151 & $=250$ & 4 & 1 & $\left(x^{5}-x\right)\left(x^{4}+2\right)$ & 800 \\
\hline 172 & $=285$ & 4 & 1 & $x(x+1)(x+2)(x-2)\left(x^{5}+2 x^{2}+1\right)$ & 912 \\
\hline 181 & $=300$ & 4 & 1 & $x(x+1)(x+2)(x-2)\left(x^{3}-x^{2}-x+2\right)\left(x^{2}+x+2\right)$ & 960 \\
\hline 199 & $=330$ & 4 & 1 & $x(x+1)(x+2)(x-2)\left(x^{5}+x^{2}-x-2\right)$ & 1056 \\
\hline
\end{tabular}


4. Further examples. In this section we construct examples of global function fields $K / \mathbb{F}_{5}$ with many rational places that are obtained by principles other than Theorem 1. In particular, we close all gaps in Table 1 in the range $23 \leq g \leq 50$. We summarize all our examples from [6], [8], and the present paper in Table 2. We list the value $g$ of the genus, a lower bound $N$ for $N_{5}(g)$, and a reference to either [6], [8], Table 1 of the present paper (abbreviated "Tb. 1"), or one of the following examples ("Ex.n" stands for Example n).

Table 2

\begin{tabular}{ccccccccccccccccc}
\hline$g$ & 1 & 2 & 3 & 4 & 5 & 6 & 7 & 8 & 9 & 10 & 11 & 12 & 13 & 14 & 15 & 16 \\
$N$ & 10 & 12 & 16 & 18 & 20 & 21 & 22 & 22 & 26 & 27 & 32 & 30 & 36 & 39 & 35 & 40 \\
$\operatorname{Ref}$ & {$[6]$} & {$[6]$} & {$[6]$} & {$[6]$} & {$[6]$} & {$[6]$} & {$[8]$} & {$[6]$} & {$[8]$} & {$[8]$} & {$[8]$} & {$[6]$} & {$[8]$} & {$[8]$} & Tb.1 & {$[8]$} \\
\hline
\end{tabular}

\begin{tabular}{ccccccccccccccccc}
\hline$g$ & 17 & 18 & 19 & 20 & 21 & 22 & 23 & 24 & 25 & 26 & 27 & 28 & 29 & 30 & 31 & 32 \\
$N$ & 42 & 32 & 45 & 30 & 50 & 51 & 55 & 46 & 52 & 45 & 52 & 54 & 56 & 58 & 72 & 62 \\
Ref & {$[8]$} & {$[8]$} & Tb.1 & [8] & Tb.1 & [8] & Tb.1 & Tb.1 & Ex.1 & Ex.2 & Tb.1 & Tb.1 & Tb.1 & Tb.1 & Ex.3 & Tb.1 \\
\hline
\end{tabular}

\begin{tabular}{lllllllllllllllll}
\hline$g$ & 33 & 34 & 35 & 36 & 37 & 38 & 39 & 40 & 41 & 42 & 43 & 44 & 45 & 46 & 47 & 48
\end{tabular}

$\begin{array}{lllllllllllllllll}N & 64 & 76 & 68 & 64 & 72 & 78 & 76 & 65 & 80 & 60 & 84 & 60 & 88 & 75 & 92 & 82\end{array}$

Ref Ex.4 Ex.5 Tb.1 Ex.6 Tb.1 Ex.7 Tb.1 Tb.1 Tb.1 Ex.8 Tb.1 Ex.9 Tb.1 Tb.1 Tb.1 Ex.10

\begin{tabular}{lllllllllllllllll}
\hline$g$ & 49 & 50 & 51 & 52 & 53 & 55 & 56 & 57 & 58 & 61 & 64 & 67 & 70 & 76 & 85 & 91
\end{tabular}

$\begin{array}{lllllllllllllllll}N & 96 & 70 & 104 & 102 & 104 & 108 & 101 & 112 & 95 & 120 & 105 & 132 & 115 & 150 & 140 & 150\end{array}$

Ref Tb.1 Ex.11 Ex.12 Tb.1 Tb.1 Tb.1 Ex.13 Tb.1 Tb.1 Tb.1 Tb.1 Tb.1 Tb.1 Tb.1 Tb.1 Tb.1

\begin{tabular}{cccccccccccccc}
\hline$g$ & 94 & 97 & 100 & 103 & 109 & 118 & 121 & 127 & 139 & 151 & 172 & 181 & 199 \\
$N$ & 155 & 160 & 165 & 170 & 180 & 195 & 200 & 210 & 230 & 250 & 285 & 300 & 330 \\
Ref & Tb.1 & Tb.1 & Tb.1 & Tb.1 & Tb.1 & Tb.1 & Tb.1 & Tb.1 & Tb.1 & Tb.1 & Tb.1 & Tb.1 & Tb.1 \\
\hline
\end{tabular}

Example 1. $g(K)=25, N(K) \geq 52$. Consider the function field $F=$ $\mathbb{F}_{5}(x, y)$ with

$$
y^{2}=x(x-1)(x-2) .
$$

Then $g(F)=1, h(F)=8$, and the place $x^{2}-2 x-2$ is inert in $F / \mathbb{F}_{5}(x)$. Let $Q$ be the unique place of $F$ lying over $x^{2}-2 x-2$. Then $\operatorname{deg}(Q)=4$. We distinguish the rational place $\infty$ of $F$ which is the unique pole of $x$, and we denote by $A$ the ring of elements of $F$ that are regular outside $\infty$. Let $E_{Q} / F$ be the narrow ray class extension of $F$ with modulus $Q$. Then

$$
\left[E_{Q}: F\right]=\left|\operatorname{Pic}_{Q}(A)\right|=h(F) \Phi_{5}(Q)=8 \cdot 624 \text {. }
$$


For $c=0,1,2 \in \mathbb{F}_{5}$ we have the principal divisors $(x-c)=2 P_{c}-2 \infty$ in $F$. Let $J$ be the subgroup of $\operatorname{Pic}_{Q}(A)$ generated by the residue classes of $P_{0}, P_{1}, P_{2}$ modulo $\mathcal{P}_{Q}(A)$. Since $P_{c}^{2}=(x-c) A$ for $c=0,1,2$ and the residue class of $x$ modulo $x^{2}-2 x-2$ generates the group $\left(\mathbb{F}_{5}[x] /\left(x^{2}-2 x-2\right)\right)^{*}$ of order 24 , the order of $J$ divides $24 \cdot 8=192$. Let $G$ be a subgroup of $\operatorname{Pic}_{Q}(A)$ with $|G|=384$ and $G \supseteq J$. Now let $K$ be the subfield of $E_{Q} / F$ fixed by $G$. Then

$$
[K: F]=\frac{8 \cdot 624}{384}=13
$$

By considering the Artin symbols, we see that $P_{0}, P_{1}, P_{2}$ split completely in $K / F$, and $\infty$ also splits completely in $K / F$, hence $N(K) \geq 52$. The only ramified place in $K / F$ is $Q$, and it is totally and tamely ramified. Thus, the Hurwitz genus formula yields $2 g(K)-2=(13-1) \cdot 4$, that is, $g(K)=25$.

Example 2. $g(K)=26, N(K) \geq 45$. Consider the function field $F=$ $\mathbb{F}_{5}(x, y)$ with

$$
y^{2}=x^{5}-x+1 \text {. }
$$

The place $x^{5}-x+1$ is totally ramified in $F / \mathbb{F}_{5}(x)$. Let $Q$ be the unique place of $F$ lying over $x^{5}-x+1$. Then $\operatorname{deg}(Q)=5$. We distinguish the rational place $\infty$ of $F$ which is the unique pole of $x$, and we denote by $A$ the ring of elements of $F$ that are regular outside $\infty$. Let $E_{M} / F$ be the narrow ray class extension of $F$ with modulus $M=Q^{2}$. Then the 5-rank of the group $\operatorname{Pic}_{M}(A) \simeq \operatorname{Gal}\left(E_{M} / F\right)$ is at least 5 by the proof of [7, Theorem 3]. For $c \in \mathbb{F}_{5}$ we have the principal divisors $(x-c)=P_{c}+P_{c}^{\prime}-2 \infty$ in $F$, with different rational places $P_{c}$ and $P_{c}^{\prime}$. The subgroup $J$ of $\operatorname{Pic}_{M}(A)$ generated by the residue classes of $P_{0}, P_{1}, P_{2}, P_{3}$ modulo $\mathcal{P}_{M}(A)$ has 5 -rank at most 4 . Thus, there exists a subgroup $G$ of $\operatorname{Pic}_{M}(A)$ with $\left[\operatorname{Pic}_{M}(A): G\right]=5$ and $G \supseteq J$.

Now let $K$ be the subfield of $E_{M} / F$ fixed by $G$. Then $[K: F]=5$. Since for each $c \in \mathbb{F}_{5}$ we have $P_{c} P_{c}^{\prime}=(x-c) A$ and

$$
(x-c)^{5^{5}-1} \equiv 1 \bmod M,
$$

we see that $G$ contains also the residue classes of $P_{0}^{\prime}, P_{1}^{\prime}, P_{2}^{\prime}, P_{3}^{\prime}$ modulo $\mathcal{P}_{M}(A)$. Therefore the places $P_{0}, P_{0}^{\prime}, P_{1}, P_{1}^{\prime}, P_{2}, P_{2}^{\prime}, P_{3}, P_{3}^{\prime}$, and $\infty$ split completely in $K / F$, hence $N(K) \geq 45$. The only ramified place in $K / F$ is $Q$, and it is totally ramified. By [11, Theorem 1 and Lemma 3] the different exponent of $Q$ in $K / F$ is 8 . Using also $g(F)=2$, we conclude from the Hurwitz genus formula that $2 g(K)-2=5 \cdot(4-2)+8 \cdot 5$, that is, $g(K)=26$.

Example 3. $g(K)=31, N(K)=72$. Let $L / \mathbb{F}_{5}$ be the function field in [6, Example 5.4] with $g(L)=4$ and $N(L)=18$. Then $\left[L: \mathbb{F}_{5}(x)\right]=9$ and all rational places of $L$ lie over the zero of $x$ or the pole of $x$ in $\mathbb{F}_{5}(x)$. The only ramified places in $L / \mathbb{F}_{5}(x)$ are those lying over $x^{2}+2$ or $x^{2}-2$, each with ramification index 3 . 
Now let $K=L(y)$ with

$$
y^{4}=\left(x^{2}+2\right)\left(x^{2}-2\right) .
$$

Then all rational places of $L$ split completely in the Kummer extension $K / L$, and so $N(K)=72$. The only ramified places in $K / L$ are those lying over $x^{2}+2$ or $x^{2}-2$, and $g(K)=31$ follows from the genus formula for Kummer extensions (see [16, Corollary III.7.4]).

EXAMPLE 4. $g(K)=33, N(K)=64, K=\mathbb{F}_{5}\left(x, y_{1}, y_{2}\right)$ with

$$
y_{1}^{4}=2-x^{4}, \quad y_{2}^{4}=2\left(x^{4}+2\right) .
$$

The places $x-1, x-2, x+1$, and $x+2$ split completely in $K / \mathbb{F}_{5}(x)$, thus $N(K)=64$. The field $L=\mathbb{F}_{5}\left(x, y_{1}\right)$ is as in [6, Example 5.3], so $g(L)=3$. The only ramified places in the Kummer extension $K / L$ are those lying over $x^{4}+2$, and $g(K)=33$ follows from the genus formula for Kummer extensions.

EXAmple 5. $g(K)=34, N(K)=76$. Consider the cyclotomic function field $E_{M}$ with modulus $M=x^{5} \in \mathbb{F}_{5}[x]$. With the rational places $P_{1}=x+1$ and $P_{2}=x-1$ of $\mathbb{F}_{5}(x)$, let $K$ be the subfield of the extension $E_{M} / \mathbb{F}_{5}(x)$ constructed in [19, Theorem 1] (see also [18, Théorème 1]). Then in the notation of [19, Theorem 1] we have

$$
s=s_{5}(2,5)=\left\lceil\log _{5} 5\right\rceil+\left\lceil\log _{5} \frac{5}{2}\right\rceil=2,
$$

and so $\left[K: \mathbb{F}_{5}(x)\right]=25$ and $N(K) \geq 25 \cdot 3+1=76$. To calculate $g(K)$, we proceed as in [19] and consider

$$
S=\left\{f \in \mathbb{F}_{5}[x]: f(x)=(x+1)^{h}(x-1)^{2 j}, h, j=0,1, \ldots\right\}
$$

and

$$
S_{r}=\left\{f \in S: x^{r} \|(f(x)-1)\right\} \quad \text { for } r=1,2, \ldots
$$

We have to determine the three least values of $r$, called $i_{1}<i_{2}<i_{3}$, for which $S_{r}$ is nonempty. It is trivial that $S_{1}$ and $S_{5}$ are nonempty. From $(x+1)^{2}(x-1)^{2}=x^{4}-2 x^{2}+1$ we conclude that $S_{2}$ is nonempty. Put

$$
S(5)=\left\{\bar{f} \in\left(\mathbb{F}_{5}[x] /\left(x^{5}\right)\right)^{*}: f \in S\right\},
$$

where $\bar{f}$ is the residue class of $f$ modulo $x^{5}$. Then $S(5)$ is generated by $\overline{x+1}$ and $\overline{x^{2}-2 x+1}$, and so $|S(5)| \leq 25$. If we had $i_{3}<5$, then $|S(5)| \geq 125$ by [19, Lemma 3], a contradiction. Therefore $i_{1}=1, i_{2}=2, i_{3}=5$. In [19, Theorem 1] we thus have $j_{1}=1$ and $j_{2}=2$, and this yields

$$
g(K)=1+\frac{1}{2} \cdot 25 \cdot 3-\frac{1}{2}\left(1+1+\frac{25-1}{4}+1\right)=34 .
$$

From $N_{5}(34) \leq 83$ it follows that $N(K)=76$. 
ExAmple 6. $g(K)=36, N(K)=64, K=\mathbb{F}_{5}\left(x, y_{1}, y_{2}, y_{3}\right)$ with

$$
y_{1}^{2}=x\left(x^{2}-2\right), \quad y_{2}^{5}-y_{2}=\frac{x^{4}-1}{y_{1}-1}, \quad y_{3}^{2}=x^{3}-2 x^{2}-x-2 .
$$

The field $L=\mathbb{F}_{5}\left(x, y_{1}, y_{2}\right)$ is as in [8, Example 4], so $g(L)=11$ and $N(L)=$ 32. All rational places of $L$ split completely in the Kummer extension $K / L$, hence $N(K)=64$. The only ramified places in $K / L$ are those lying over $x^{3}-2 x^{2}-x-2$, and $g(K)=36$ follows from the genus formula for Kummer extensions.

ExAmple 7. $g(K)=38, N(K)=78$. Consider the cyclotomic function field $E_{Q}$ with $Q=x^{4}-2 \in \mathbb{F}_{5}[x]$. Let $G$ be the cyclic subgroup of $\left(\mathbb{F}_{5}[x] /\left(x^{4}-2\right)\right)^{*} \simeq \operatorname{Gal}\left(E_{Q} / \mathbb{F}_{5}(x)\right)$ generated by the residue class of $x$ modulo $x^{4}-2$. Then $|G|=16$. Now let $K$ be the subfield of $E_{Q} / \mathbb{F}_{5}(x)$ fixed by $G$. Then $\left[K: \mathbb{F}_{5}(x)\right]=39$. The zero of $x$ and the pole of $x$ in $\mathbb{F}_{5}(x)$ split completely in $K / \mathbb{F}_{5}(x)$, thus $N(K) \geq 78$. The only ramified place in $K / \mathbb{F}_{5}(x)$ is $Q$, and it is totally and tamely ramified. Therefore the Hurwitz genus formula yields $2 g(K)-2=39 \cdot(-2)+(39-1) \cdot 4$, that is, $g(K)=38$. From $N_{5}(38) \leq 91$ it follows that $N(K)=78$.

Example 8. $g(K)=42, N(K)=60, K=\mathbb{F}_{5}\left(x, y_{1}, y_{2}\right)$ with

$$
y_{1}^{2}=\left(x^{2}+2\right)\left(x^{4}-2 x^{2}-2\right), \quad y_{2}^{5}-y_{2}=\frac{x^{5}-x}{\left(x^{2}+2\right)\left(x^{4}-2 x^{2}-2\right)} .
$$

The field $L=\mathbb{F}_{5}\left(x, y_{1}\right)$ is as in [6, Example 5.2], so $g(L)=2$ and $N(L)=12$. All rational places of $L$ split completely in the Artin-Schreier extension $K / L$, hence $N(K)=60$. The only ramified places in $K / L$ are the unique place of $L$ of degree 2 lying over $x^{2}+2$ and the unique place of $L$ of degree 4 lying over $x^{4}-2 x^{2}-2$, thus $g(K)=42$ follows from the genus formula for Artin-Schreier extensions (see [16, Proposition III.7.8]).

ExAmple 9. $g(K)=44, N(K)=60, K=\mathbb{F}_{5}\left(x, y_{1}, y_{2}\right)$ with

$$
y_{1}^{5}-y_{1}=\frac{x^{5}-x}{\left(x^{2}+2\right)^{3}}, \quad y_{2}^{2}=\left(x^{2}+2\right)\left(x^{8}-x^{4}-x^{2}-2\right) .
$$

The field $L=\mathbb{F}_{5}\left(x, y_{1}\right)$ is as in [6, Example 5.12A], so $g(L)=12$ and $N(L)=30$. All rational places of $L$ split completely in the Kummer extension $K / L$, hence $N(K)=60$. The only ramified places in $K / L$ are the unique place of $L$ of degree 2 lying over $x^{2}+2$ and the places of $L$ lying over $x^{8}-x^{4}-x^{2}-2$, thus $g(K)=44$ follows from the genus formula for Kummer extensions.

EXAmPle 10. $g(K)=48, N(K)=82, K=\mathbb{F}_{5}\left(x, y_{1}, y_{2}, y_{3}\right)$ with

$$
y_{1}^{2}=x\left(x^{2}-2\right), \quad y_{2}^{5}-y_{2}=\frac{x^{4}-1}{y_{1}}, \quad y_{3}^{2}=x^{3}-2 x^{2}-x-2 .
$$


The field $L=\mathbb{F}_{5}\left(x, y_{1}, y_{2}\right)$ is as in [8, Example 9], so $g(L)=17$ and $N(L)=42$. All rational places of $L$, except the unique place of $L$ lying over $x$, split completely in the Kummer extension $K / L$, hence $N(K)=82$. The only ramified places in $K / L$ are those lying over $x^{3}-2 x^{2}-x-2$, and $g(K)=48$ follows from the genus formula for Kummer extensions.

EXAMPLE 11. $g(K)=50, N(K)=70$. Let $L / \mathbb{F}_{5}$ be the function field in Table 1 with $g(L)=15$ and $N(L)=35$. By the construction in the proof of Theorem 1 we have $\left[L: \mathbb{F}_{5}(x)\right]=14$, and the rational places of $L$ lie over $x, x+1, x+2, x-1$ or the pole of $x$, with each rational place of $L$ having ramification index 2 over $\mathbb{F}_{5}(x)$. Now let $K=L(z)$ with

$$
z^{2}=x^{3}+2 x^{2}-x-1 \text {. }
$$

Then all rational places of $L$ split completely in the Kummer extension $K / L$, hence $N(K)=70$. The only ramified places in $K / L$ are those lying over $x^{3}+2 x^{2}-x-1$, and $g(K)=50$ follows from the genus formula for Kummer extensions.

EXAMPLE 12. $g(K)=51, N(K)=104$. Let $E_{Q} / F$ be the same narrow ray class extension as in Example 1 and let $J$ be the same subgroup of $\operatorname{Pic}_{Q}(A)$ as in Example 1. Let $G$ be a subgroup of $\operatorname{Pic}_{Q}(A)$ with $|G|=$ 192 and $G \supseteq J$. Now let $K$ be the subfield of $E_{Q} / F$ fixed by $G$. Then $[K: F]=26$. As in Example 1 we see that the places $P_{0}, P_{1}, P_{2}$, and $\infty$ split completely in $K / F$, hence $N(K) \geq 104$. The only ramified place in $K / F$ is $Q$, and it is totally and tamely ramified. Thus, the Hurwitz genus formula yields $2 g(K)-2=(26-1) \cdot 4$, that is, $g(K)=51$. From $N_{5}(51) \leq 115$ it follows that $N(K)=104$.

EXAmPle 13. $g(K)=56, N(K)=101$. Consider the cyclotomic function field $E_{M}$ with modulus $M=x^{7} \in \mathbb{F}_{5}[x]$. With the rational places $P_{1}=x+1, P_{2}=x-1$, and $P_{3}=x+2$, let $K$ be the subfield of the extension $E_{M} / \mathbb{F}_{5}(x)$ constructed in [19, Theorem 1] (see also [18, Théorème 1]). Then in the notation of [19, Theorem 1] we have

$$
s=s_{5}(3,7)=\left\lceil\log _{5} 7\right\rceil+\left\lceil\log _{5} \frac{7}{2}\right\rceil+\left\lceil\log _{5} \frac{7}{3}\right\rceil=4,
$$

and so $\left[K: \mathbb{F}_{5}(x)\right]=25$ and $N(K) \geq 25 \cdot 4+1=101$. To calculate $g(K)$, we proceed as in [19] and consider

$S=\left\{f \in \mathbb{F}_{5}[x]: f(0)=1, f(x)=(x+1)^{h}(x-1)^{j}(x+2)^{k}, h, j, k=0,1, \ldots\right\}$

and

$$
S_{r}=\left\{f \in S: x^{r} \|(f(x)-1)\right\} \quad \text { for } r=1,2, \ldots
$$

We have to obtain information on the five least values of $r$, called $i_{1}<i_{2}<$ $i_{3}<i_{4}<i_{5}$, for which $S_{r}$ is nonempty. It is trivial that $S_{1}$ and $S_{5}$ are nonempty. From $(x+1)^{2}(x-1)^{2}=x^{4}-2 x^{2}+1$ we conclude that $S_{2}$ is 
nonempty, and from

$$
(x+1)(x-1)^{8}(x+2)^{4}=x^{13}+\ldots+2 x^{3}+1
$$

we conclude that $S_{3}$ is nonempty. Therefore $i_{1}=1, i_{2}=2, i_{3}=3$. Put

$$
S(5)=\left\{\bar{f} \in\left(\mathbb{F}_{5}[x] /\left(x^{5}\right)\right)^{*}: f \in S\right\},
$$

where $\bar{f}$ is the residue class of $f$ modulo $x^{5}$. Then $S(5)$ is generated by $\overline{1+x}$, $\overline{1-x}$, and $\overline{1-2 x}$, and so $|S(5)| \leq 5^{3}$. If we had $i_{4}=4$, then $|S(5)|=5^{4}$ by [19, Lemma 3], a contradiction. Therefore $i_{4}=5$. Put

$$
S(7)=\left\{\overline{\bar{f}} \in\left(\mathbb{F}_{5}[x] /\left(x^{7}\right)\right)^{*}: f \in S\right\},
$$

where $\overline{\bar{f}}$ is the residue class of $f$ modulo $x^{7}$. Then $S(7)$ is generated by $\overline{\overline{1+x}}, \overline{\overline{1-x}}$, and $\overline{\overline{1-2 x}}$. Since $S(7)$ is contained in the 5-Sylow subgroup of $\left(\mathbb{F}_{5}[x] /\left(x^{7}\right)\right)^{*}$, it follows from $\left[19\right.$, Lemma 4(ii)] that $|S(7)| \leq 5^{s}=5^{4}$. If we had $i_{5}=6$, then $|S(7)|=5^{5}$ by [19, Lemma 3], a contradiction. Therefore $i_{5} \geq 7$. In [19, Theorem 1] we thus have $j_{1}=1, j_{2}=2, j_{3}=3, j_{4}=5$, and this yields

$$
g(K)=1+\frac{1}{2} \cdot 25 \cdot 5-\frac{1}{2}\left(1+1+1+5+\frac{25-1}{4}+1\right)=56 .
$$

From $N_{5}(56) \leq 125$ it follows that $N(K)=101$.

\section{References}

[1] A. Garcia and H. Stichtenoth, Algebraic function fields over finite fields with many rational places, IEEE Trans. Inform. Theory 41 (1995), 1548-1563.

[2] D. Goss, Basic Structures of Function Field Arithmetic, Springer, Berlin, 1996.

[3] D. R. Hayes, Explicit class field theory for rational function fields, Trans. Amer. Math. Soc. 189 (1974), 77-91.

[4] - A brief introduction to Drinfeld modules, in: The Arithmetic of Function Fields, D. Goss, D. R. Hayes, and M. I. Rosen (eds.), de Gruyter, Berlin, 1992, 1-32.

[5] H. Niederreiter and C. P. Xing, Quasirandom points and global function fields, in: Finite Fields and Applications, S. Cohen and H. Niederreiter (eds.), Cambridge Univ. Press, Cambridge, 1996, 269-296.

[6] -, - Cyclotomic function fields, Hilbert class fields, and global function fields with many rational places, Acta Arith. 79 (1997), 59-76.

[7] - - - Drinfeld modules of rank 1 and algebraic curves with many rational points. II, ibid. 81 (1997), 81-100.

[8] - - - Global function fields with many rational places over the quinary field, Demonstratio Math. 30 (1997), 919-930.

[9] —, - , The algebraic-geometry approach to low-discrepancy sequences, in: Monte Carlo and Quasi-Monte Carlo Methods 1996, H. Niederreiter et al. (eds.), Lecture Notes in Statist. 127, Springer, New York, 1998, 139-160.

[10] - - - Algebraic curves over finite fields with many rational points, in: Number Theory, K. Győry, A. Pethő, and V. T. Sós (eds.), de Gruyter, Berlin, 1998, 423-443. 
[11] H. Niederreiter and C. P. Xing, Global function fields with many rational places and their applications, in: Proc. Finite Fields Conf. (Waterloo, 1997), Contemp. Math., Amer. Math. Soc., Providence, to appear.

[12] - - - Nets, $(t, s)$-sequences, and algebraic geometry, in: Pseudo- and QuasiRandom Point Sets, P. Hellekalek and G. Larcher (eds.), Lecture Notes in Statist., Springer, New York, to appear.

[13] O. Pretzel, Codes and Algebraic Curves, Oxford Univ. Press, Oxford, 1998.

[14] M. Rosen, The Hilbert class field in function fields, Exposition. Math. 5 (1987), 365-378.

[15] J.-P. Serre, Sur le nombre des points rationnels d'une courbe algébrique sur un corps fini, C. R. Acad. Sci. Paris Sér. I Math. 296 (1983), 397-402.

[16] H. Stichtenoth, Algebraic Function Fields and Codes, Springer, Berlin, 1993.

[17] G. van der Geer and M. van der Vlugt, How to construct curves over finite fields with many points, in: Arithmetic Geometry, F. Catanese (ed.), Cambridge Univ. Press, Cambridge, 1997, 169-189.

[18] C. P. Xing and H. Niederreiter, Modules de Drinfeld et courbes algébriques ayant beaucoup de points rationnels, C. R. Acad. Sci. Paris Sér. I Math. 322 (1996), 651-654.

[19] - - - Drinfeld modules of rank 1 and algebraic curves with many rational points, Monatsh. Math., to appear.

Institut für Informationsverarbeitung

Österreichische Akademie

der Wissenschaften

Sonnenfelsgasse 19

A-1010 Wien, Austria

E-mail: niederreiter@oeaw.ac.at
Department of Information Systems and Computer Science

The National University of Singapore Lower Kent Ridge Road Singapore 119260

E-mail: xingcp@comp.nus.edu.sg 